\title{
Performance Analysis of MIMO MC-DS/CDMA System Using Chaotic Spreading Sequence
}

\author{
V.Nagarajan and P. Dananjayan
}

\begin{abstract}
${ }^{1}$ Abstract - This paper presents a novel chaotic spreading sequence for multiple input multiple output multi-carrier direct sequence code division multiple access (MIMO MCDS/CDMA) systems. The effect of multiple access interference can be mitigated by choosing the spreading sequences with appropriate cross-correlation properties. The performance of the system is analysed in multiuser scenario with the aid of simulation. The simulation results show that the proposed chaotic code spreading approach achieves a significant improvement in system utility and combats multiple access interference (MAI). The analysis reveals that the proposed system achieves significant performance improvement compared to Walsh Hadamard spreading code in MIMO MCDS/CDMA systems.
\end{abstract}

Index Terms-MC-DS/CDMA, Chaotic code, MIMO, Spreading sequence, MAI

\section{INTRODUCTION}

With increasing demands on current wireless systems put forth by high-speed packet data and multimedia streaming services, technologies that will deliver increased capacity has enamored researchers in recent years. While a prolific literature is available on increasing user data rate, spreading gain, they do so at the expense of reducing the total system throughput. A true high-speed multiuser wireless system can only be achieved through an increase in system spectral efficiency, measured in bits per second per Hertz per sector. The wireless MIMO [1,2]communication systems seek to achieve capacities close to Shannon limit by employing multiple transmit and receive antenna, with advanced spacetime signal processing techniques. Multicarrier spread spectrum techniques offer tremendous scope for next generation (4G) high-speed wireless technologies, where spectral efficiency and flexibility are important.

To support multiple users, the multicarrier transmission technique can be combined with a CDMA scheme. Due to wide bandwidth requirement of wireless communication system, the combination of MIMO MC-DS/CDMA with spreading in both time and frequency domain has recently attracted a lot of interest in wireless communication and provides an efficient approach to reduce the chip rate and the spreading code length [2-6].

The major challenge in MIMO MC-DS/CDMA is stringent power control. A new approach to the power control problem in wireless systems based on an economic model has been suggested $[4,5]$. In this model, service preferences for each user are represented by a utility

\footnotetext{
${ }^{1}$ Department of Electronics and Communication Engineering Pondicherry Engineering College, Pondicherry-605014, India pdananjayan@rediffmail.com
}

function, which quantifies the level of satisfaction a user gets in using the system resources. Game theoretic methods are applied to determine the effect of power control under this new model. Game theory is a powerful tool in modeling interactions between self-interested users and predicting their choice of strategies. Each player in the game maximizes some function of utility in a distributed fashion. [7,8]. The game settles at Nash equilibrium if one exits. Since users act selfishly, the equilibrium point is not necessarily the best operating point from a social point of view $[9,10]$. Pricing the system resources appears to be a powerful tool for achieving a more socially desirable result. A non- cooperative power control game with pricing (NPGP) in MC-DS/CDMA has been investigated in [7, 8] and the existence of nash equilibrium and corresponding sub-carrier allocation has also been addressed.

A classical set of spreading sequences used in DSCDMA systems are the binary sequences generated by linear feedback shift register (LFSR) schemes [11]. Generation of fairly good set of Gold, Kasami and Walsh hadamard sequences require a large set dimension and period, which are generally limited by the LFSR polynomial degree [10,12-14]. This puts forth the need for optimal codes, which is the prime motive of this work. These spreading sequences should possess minimal crosscorrelation values to reduce the multiple access interference (MAI) [10].

When more users are active, the performance degradation due to MAI becomes more obvious. However the total utility improvement relies on the ability of the receiver to detect the desired signal in the presence of interference. This to a great extent relies on the good cross correlation properties of the spreading codes (sequences). Therefore, a successful implementation of MIMO MCDS/CDMA systems strongly demands for spreading sequences that are capable of injecting minimal interference. The dependence of spread sequences on CDMA system performance is exhaustively discussed in [6].This work aims at employing the non-cooperative power control game with pricing (NPGP) with chaotic sequences such that the MAI is effectively reduced in a MC DS/CDMA environment comparing with Walsh spreading sequences. Interference parameter is one form of optimization criterion that is necessary to minimize MAI.

The rest of the paper is organized as follows: Section II illustrates the MC-DS/CDMA system model. Section III deals with chaotic codes and its comparison with conventional codes. Section IV introduces a pricing strategy to improve the power efficiency and overall system utility. Section V presents simulation results to demonstrate 
the performance improvement resulting from this approach and finally the conclusion is given in Section VI.

\section{Mimo MC-Ds/CDMA SYSTEM MODEL}

Fig.1 illustrates the transmitter of the MIMO MC DS/CDMA system employing both Time and Frequency domain, i.e. TF-domain spreading [13] the $\mathrm{k}^{\text {th }}$ user. At the transmitter, the binary data stream $b_{k}(t)$ is first directsequence (DS) spread using T-domain signature sequence $a_{k}(t)$. The T-domain DS spread signal is divided into $M$ parallel branches, where each branch-signal is multiplied by a corresponding chip value of $\mathrm{F}$-domain spreading sequence $\mathrm{C}_{\mathrm{k}}=\left\{\mathrm{C}_{\mathrm{k}}[1], \mathrm{C}_{\mathrm{k}}[2], \mathrm{C}_{\mathrm{k}}[3] \ldots . \mathrm{C}_{\mathrm{k}}[\mathrm{M}]\right\}^{\mathrm{T}}$ of length $\mathrm{M}$. Following F-domain spreading, each of the $\mathrm{M}$ branch signals modulates a sub carrier frequency using binary phase shift keying (BPSK). Then, the $M$ numbers of subcarriermodulated substreams are added in order to form the transmitted signal. Hence, the transmitted MIMO MC DS/CDMA signal of $\mathrm{s}(\mathrm{t})$ user $\mathrm{k}$ can be expressed as

$$
S_{k}(t)=\sum_{k=1}^{\min \left\{M_{t,} M_{r}\right\}}\left(\sqrt{\frac{2 P_{K}}{M}} \sum_{m=1}^{M} b_{k}(t) a_{k}(t) c_{k}[m] \cos \left(w_{m} t\right), k=1,2,3 \ldots . K\right)
$$

where $P_{k}$ represents the transmitted power of the $k^{\text {th }}$ user and $\left\{\mathrm{w}_{\mathrm{m}}\right\}$, represents the subcarrier frequency set. $\mathrm{M}_{\mathrm{t}}$ and $\mathrm{M}_{\mathrm{r}}$ are the number of transmit and receive antennas respectively. The binary data stream $b_{k}(t)$ consists of sequence of mutually independent rectangular pulses $\mathrm{P}_{\mathrm{Tb}}$ of duration $\mathrm{T}_{\mathrm{b}}$ and amplitude +1 or -1 with equal probability. The spreading sequence, $a_{k}(t)$ denotes the $T$-domain spreading waveform of the $k^{\text {th }}$ user with spreading factor $N=T_{b} / T_{C}$, represents the number of chips per bit-duration. It is assumed that the subcarrier signals are orthogonal and their spectral main-lobes of are not overlapping with each other. The received signal can be expressed as

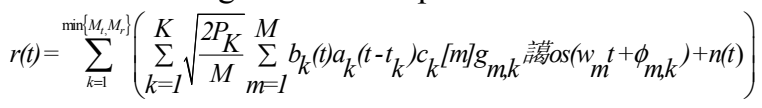

where $n(t)$ represents the AWGN having zero mean and double-sided power spectral density of $\mathrm{N}_{0} / 2$. As shown in Fig.2 and Eq.(1), each TF-domain spread MC DS-CDMA signal is identified with the aid of two spreading sequences.

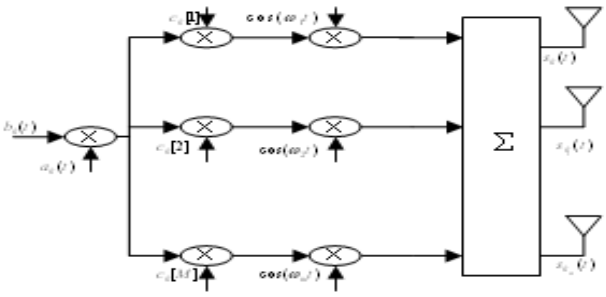

Fig 1. Transmitter model of MIMO MC DS-CDMA

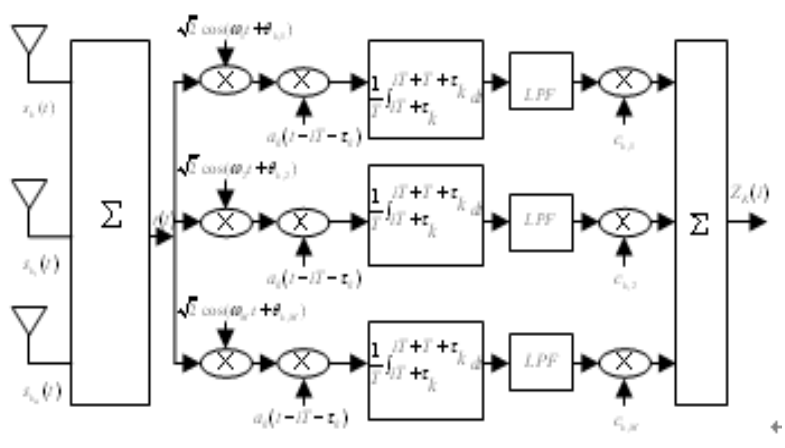

Fig 2. Receiver model of MIMO MC DS-CDMA

\section{CHOATIC SPREADING CODES}

The pseudo-noise sequences such as Gold sequences and Walsh hadamard sequences are the most popular spreading sequences that have good correlation properties, limited security and are reconstructed by linear regression attack for their short linear complexity [12]. A chaotic sequence generator can visit an infinite number of states in a deterministic manner and therefore produce a sequence which never repeats itself [14]. The designer has the flexibility in choosing the spreading gain as the sequences can be truncated to any length. Many authors have shown that chaotic spreading sequences can be used as an inexpensive alternative to the LFSR [2,3]. However, search for the best set of codes contributing reduced MAI is still a severe requirement of future MC- DS/CDMA systems. Generation of good set of sequences demands for large set dimension, period and limited privacy. To overcome these limitations, new chaotic spreading codes, is used in this work. Instead of using other spreading codes in MIMO MC DS-CDMA, chaotic code can produce good result in terms of utility as well as mitigating MAI. A single system described by discrete chaotic map generates large number of distinct chaotic sequences, each sequence being uniquely specified by its initial value. This dependency on initial state and non-linear characteristic of discrete map makes the DS-CDMA system highly secure. A chaotic map is a dynamic discrete-time continuous-value equation that describes the relation between the present and next value of chaotic system. Let $X_{n+1}$ and $X_{n}$ be successive iterations of the output $\mathrm{X}$ and $\mathrm{M}$ is the forward transformation mapping function. The general form of multidimensional chaotic map is $X_{n+1}=M\left(X_{n}, X_{n-1} \ldots\right.$ $\left.X_{n-m}\right)$.

A simple logistic map is given as

$X_{n+1}=\mu X_{n}\left(1-X_{n}\right), 0<X_{n}<1$, and $1 \leq \mu \leq 4$

where $\mu$ is the bifurcation parameter and the system exhibits a great variety of dynamics depending on the value of $\mu, \quad(3.6 \leq \mu \leq 6)$. 


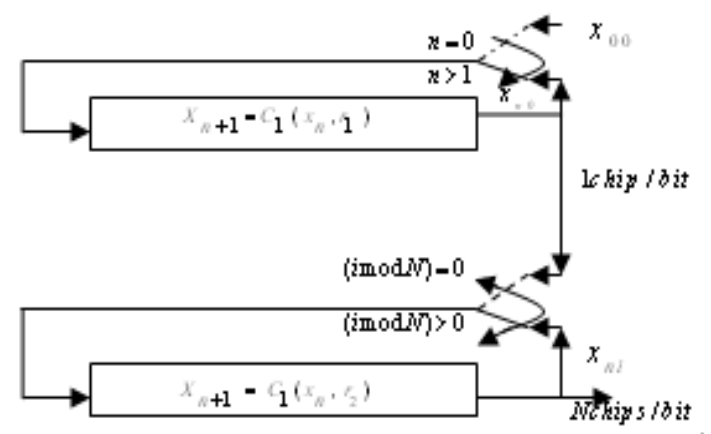

Fig. 3 Generation of chaotic sequences

Using logistic map the chaotic spreading sequences for MC DS CDMA system is generated. After assigning different initial condition to each user, the chaotic map is started with the initial condition of the intended receiver and is iterated repeatedly to generate multiple codes. It is assumed that the transmitter and the intended receiver have agreed upon a starting point, $\mathrm{x}_{00}$ and two chaotic maps, $\mathrm{C}_{1}(\mathrm{x}$, $\left.r_{1}\right)$ and $C_{2}\left(x, r_{2}\right)$ with their corresponding bifurcation parameters, $r_{1}$ and $r_{2}$. The chaotic maps and their bifurcation parameters may or may not be the same and their uniqueness among the different pairs of transmitters and receivers is not necessary. In Fig. $3, \mathrm{x}_{00}$ initiates a chaotic sequences $\mathrm{X}_{0}=\left\{\mathrm{x}_{\mathrm{n} 0}: \mathrm{n}=0,1,2, \ldots \ldots\right\}$ through the chaotic map $\mathrm{C}_{1}\left(\mathrm{x}, \mathrm{r}_{1}\right)$. The elements of the sequence are then used to generate the sequences $\mathrm{S}_{\mathrm{n}}=\left\{\mathrm{x}_{\mathrm{ni}}: \mathrm{i}=0,1,2 \ldots\right\}, \mathrm{n}=0,1,2 \ldots$ through the chaotic map $\mathrm{C}_{2}\left(\mathrm{x}, \mathrm{r}_{2}\right)$. The sequences $\mathrm{S}_{\mathrm{n}}$, so obtained are the spreading sequences to be used for each data bit. The receiver regenerates the sequence $S_{n}$ exactly in the same manner as the transmitter does. Every receiver will be assigned distinct $\mathrm{x}_{00}, \mathrm{C}_{1}\left(\mathrm{x}, \mathrm{r}_{1}\right), \mathrm{C}_{2}\left(\mathrm{x}, \mathrm{r}_{2}\right), \mathrm{r}_{1}$ and $\mathrm{r}_{2}$, and therefore, the resulting spreading sequences for each receiver in a multiple-access communication system will be completely independent and uncorrelated.

\section{Pricing Strategy To InCREASE Entire System UTILITY}

In the non cooperative power control game ( NPG), each terminal aims to maximize its own utility by adjusting its own power, but it ignores the cost (or harm) it imposes on other terminals by the interference it generates. The selfoptimizing behaviour of an individual terminal is said to create an externality when it degrades the quality for every other terminal in the system. Among the many ways to deal with externalities, pricing (or taxation) has been used as an effective tool both by economists and researchers in the field of computer networks. Typically, pricing is motivated by two different objectives. First it generates revenue for the system and secondly it encourages players to use system resources more efficiently. Pricing does not refer to monetary incentives, but rather refers to a control signal to motivate users to adopt a social behavior. An efficient pricing mechanism makes decentralized decisions compatible with overall system efficiency by encouraging efficient sharing of resources rather than the aggressive competition of the purely non cooperative game. A pricing policy is called incentive compatible if pricing enforces a Nash equilibrium that improves social welfare. A social welfare is defined as the sum of utilities. In order to improve the equilibrium utilities of NPG in the Pareto sense, the usage-based pricing schemes has been introduced in [12].Through pricing, system performance can be increased by implicitly inducing cooperation and the non cooperative nature of the resulting power control solution had been maintained. An efficient pricing scheme should be tailored for the problem at hand. Within the context of a resource allocation problem for a wireless system, the resource being shared is the radio environment and the resource usage is determined by terminal's transmit power. Although the Nash equilibrium provides a self-optimizing power control solution for individual user, it is not necessarily the best operating point for the whole system. That is, there exist the other power solutions to make the utilities of all the users greater than those at the Nash equilibrium. In order to make efficient use of system resource, non cooperative power control game with pricing (NPGP) is introduced to force each user to efficiently share rather than aggressively approaches the system resource.

The utility function of $k^{\text {th }}$ user for a MIMO MC$\mathrm{DS} / \mathrm{CDMA}$ is defined as the ratio of the total throughput to the total transmits power off all sub-carriers is given as

$$
u_{k}^{M}=\sum_{k=1}^{\min \left\{M_{1}, M_{r}\right\}}\left(\frac{L}{D} R_{k} \frac{f\left(\gamma_{k}\right)}{\sum_{m=1}^{M} P_{k, m}}\right)
$$

where $L$ and $D$ are the number of information bits and the number of bits in a packet, respectively, $R_{k}$ is the transmission rate for user $k, P_{k}$ is the transmit power of user $k$, and $f\left(\gamma_{k}\right)$ is the efficiency function for the transmission of user $k$. The utility function of Eq. (4) with pricing is reformulated as

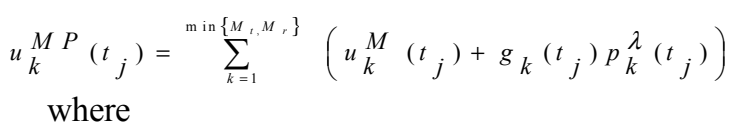

$\mathrm{u}_{\mathrm{k}}{ }^{M P}\left(\mathrm{t}_{\mathrm{j}}\right)$ is utility function with pricing

$\mathrm{u}_{\mathrm{k}}{ }^{\mathrm{M}}\left(\mathrm{t}_{\mathrm{j}}\right)$ is traditional utility function

$\mathrm{g}_{\mathrm{k}}\left(\mathrm{t}_{\mathrm{j}}\right) \quad$ is set of updated instances for all the users $P_{\mathrm{k}}^{\lambda}\left(\mathrm{t}_{\mathrm{j}}\right)$ is transmitted power

In the proposed NPG with pricing each user is forced to effectively share the system resource rather than by an aggressive approach of late many pricing policies is in use and usage based policies is one of the common policies adapted in MC DSCDMA system . In this scheme each user pays the penalty which is proportional to the transmit power. In our approach a new utility function with a pricing factor given by the equation (5) to address the problem of individual utility and interference to the other users that maximizes the total utility is proposed. These eliminate the problem of picking up the good pricing factor in the conventional approach which involves computational complexity has been eliminated.

\section{NUMERICAL RESULTS}

For comparison of chaotic and Walsh spreading code, it 
is assumed that the number of information bits per frame $L$ $=64$, the total number of bits per frame $D=80$; the transmission rate for each user $\mathrm{R}_{\mathrm{K}}$ is $10^{5} \mathrm{bits} / \mathrm{s}$; the total transmit power $\mathrm{P}_{\max }$ is limited by $6 \mathrm{~W}$ and $\mathrm{P}_{\min }=0.1 \mathrm{~W}$ with $\mathrm{M}_{\mathrm{t}}=2$ and $\mathrm{M}_{\mathrm{r}}=2$ for the analysis

Fig.4 expounds the comparisons for Walsh Hadamard and chaotic code for fifty user in terms of utility function. The system employs Walsh code and chaotic of length 64 bits . Walsh code yields around $10 \times 10^{6}$ utility where as chaotic code yields $10.5 \times 10^{6}$. This shows five percent improvement in the total utility has been achieved by the use of chaotic sequence. NPGP sets up certain cooperation among terminals via the pricing strategy, and each terminal tries to increase its own utility and reduces the interference to other users as well. Although Nash equilibriums are the best operating points to increase the traditional utility function $\mathrm{u}^{\mathrm{M}}{ }_{\mathrm{k}}$, each player in NPG cares about their own utility, and may generate significant interference to other users. Therefore, considerable improvement is achieved by NPGP with chaotic sequence when many users are active, mitigating MAI. Fig.4 also elucidates plot for scheme without pricing. It can be discerned that this scheme does not perform well in terms of total utility function compared to scheme with pricing.

The total utility for chaotic and Walsh code is compared with different user in figs. 5 and 6 by varying the noise power from $10^{-6}$ to $10^{-2}$. It is observed that, when noise power is around $10^{-3}$, the utility start to decrease drastically due to increase in noise power. But there is a remarkable improvement in utility factor by using chaotic code when the noise power level increases. It is noted that chaotic code outperforms by $9 \%$ compared to Walsh hadmard code in terms of utility factor and is excellent in mitigating MAI, because of its good cross correlation properties, which in turn results in capacity enhancement of the system.

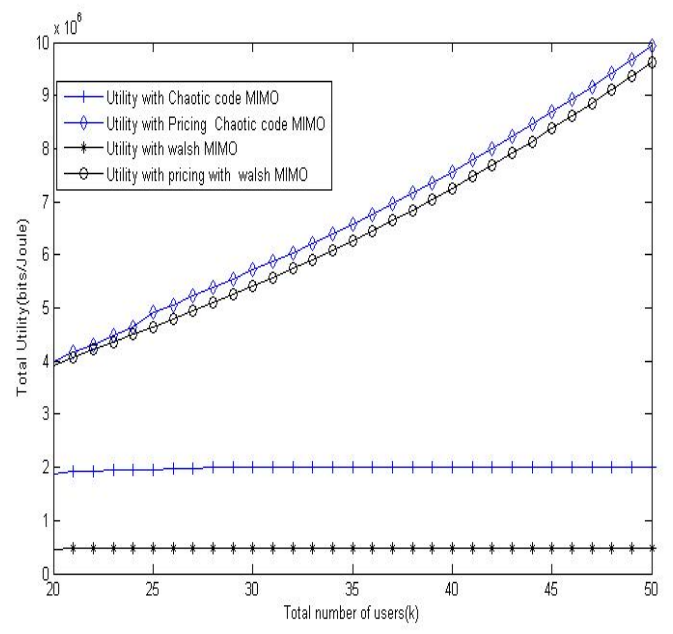

Fig.4. Total utility vs total number of users $\mathrm{K}$

Furthermore, when the noise power at the receiver side increases, the SNR $\left(\gamma_{\mathrm{k}}\right)$ and efficiency function $f\left(\gamma_{k}\right)$ decreases. Thus, less utility is obtained with increase in $\sigma_{\mathrm{N}}^{2}$.

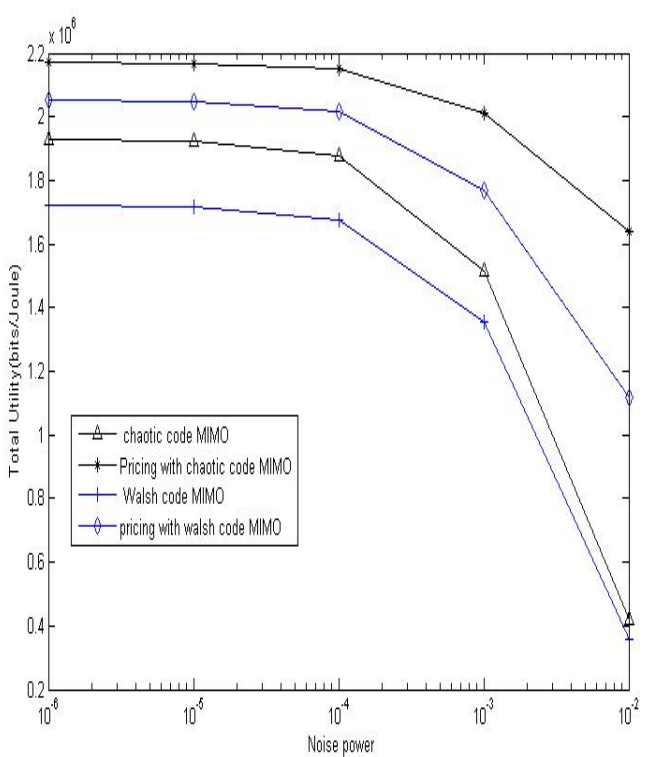

Fig.5. Total utility vs noise power

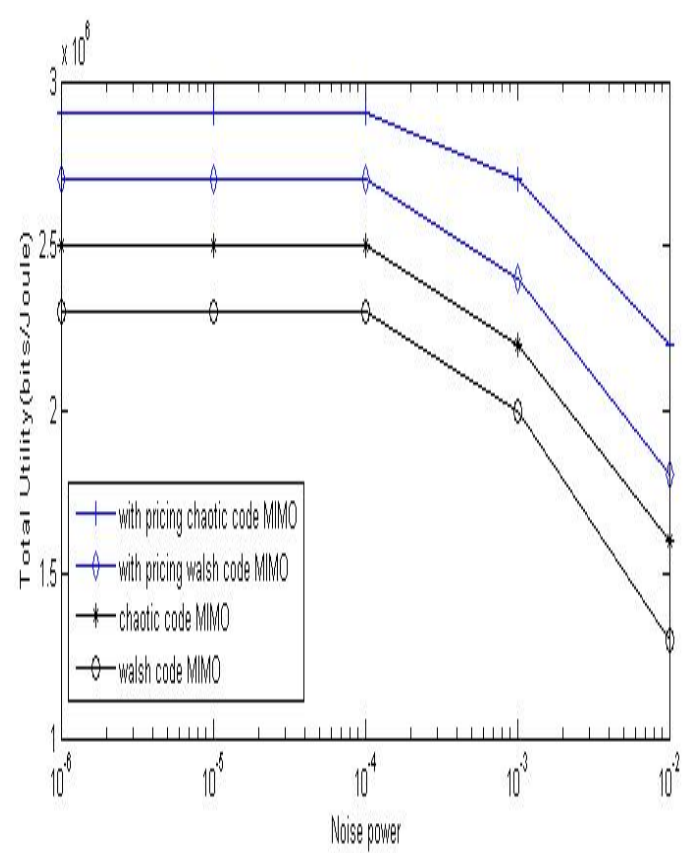

Fig.6. Total utility vs noise power

When the noise power is limited to $10^{-4}$, the utility factor is appreciable. On the other hand, when noise power increases, the efficiency function $f\left(\gamma_{k}\right)$ decreases significantly. For instance the utility factor drops drastically from $2.7 \times 10^{6}$ bits /joules to $1.8 \times 10^{6}$ bits/joules for conventional NPGP, when the noise power increases from $10^{-4}$ to $10^{-2}$. Whereas for the proposed NPGP with chaotic sequence, the utility factor does not decrease drastically for a noise power of $10^{-2}$ and is around $2.2 \times 10^{6}$ bits/joules which is about $8 \%$ increase in total utility. This is due to the fact that the terminals in the modified NPGP carefully manage the transmit power to increase individual utility and combat MAI as well, since modified NPGP can tolerate higher noise power than conventional NPGP. Also the total utility versus noise power for pricing scheme is taken into consideration for our analysis. Numerical results divulge that the pricing scheme outperforms the scheme without pricing in terms 
of utility.

\section{CONCLUSION}

In this paper chaotic spreading codes for MIMO MC DS/CDMA has been considered for the analysis. A NPG algorithm based on pricing performs better by effectively mitigating the MAI in MIMO MC DS-CDMA system. Also the use of chaotic codes performs better than the system with classical codes in exterminating MAI. Numerical results of our analysis shows that employing chaotic code with good cross correlation properties can minimizes the interference in a multiuser MIMO MC DS CDMA system. The proposed scheme also achieves significant improvement in the utility factor compare to the conventional system without pricing.

\section{REFERENCES}

[1] L.Yang W. Hua, and L. Hanzo, "Multi-user Detection in Multicarrier CDMA Systems Employing Both Time-Domain and Frequency-Domain Spreading”, Proc. of PIMRC'03, vol. 2, pp. 18401844, Sept. 2003.

[2] Hua Wei, Lie-Liang Yang and Lajos Hanzo, "Time and frequency domain spreading assisted MC DS-CDMA using interference rejection spreading codes for quasi-synchronous communications" Proc of IEEE Vehicular Technology Conference, pp. 389-393, Sept. 2004.

[3] L.Yang and L. Hanzo, "Performance of Broadband Multi-carrier DSCDMA Using Space-Time Spreading-Assisted Transmit Diversity", IEEE Trans. Wireless Comm., vol. 4, no. 3, pp. 885-894, May 2005

[4] E.Sourour and M. Nakagawa, "Performance of Orthogonal MultiCarrier CDMA in Multi-Path Fading Channels", IEEE Trans. Comm., vol. 44, no. 3, pp. 356-367, Mar. 1996.

[5] S.Kondo and L. B. Milstein, "Performance of Multi-carrier DS CDMA Systems", IEEE Trans. Comm., vol.44,no.2, pp. 238-246, Feb. 1996.

[6] Lin Fang and Rui J.P.de Figueiredo, "A Game-Theoretic Approach to Utility based Power Control in Multi-Carrier DS/CDMA Systems", Proc. of IEEE CCNC, pp.155-159,Feb. 2007.

[7] V.Shah, N. B. Mandayam, and D. J. Goodman, "Power Control for Wireless Data Based on Utility and Pricing", Proc. of PIMRC, pp. 1427-1432, 1998.

[8] C.U.Saraydar, Mandayam N. B, and. Goodman .D. J, "Efficient Power Control via Pricing in Wireless Data Networks", IEEE Trans on Communication,vol. 50, no. 2, pp. 291-303, Feb. 2002.

[9] F.Meshkati., M. Chiang, S.C. Schwartz and H.V. Poor, "A NonCooperative Power Control Game for Multi-Carrier CDMA Systems",Proc of IEEE Wireless Communications and Networking Conference, Vol. 1,pp .606 - 611, Mar. 2005.

[10] S.Kondo and L.B. Milstein, "On the Use of Multi carrier Direct Sequence Spread Spectrum Systems", MILCOM 93, Boston, MA, pp. 52-56, Oct. 1993.

[11] Ling cong and Li Shaoqjan, "Chaotic Spreading Sequences with Multiple Access Performance Better Than Random Sequences", IEEE Transaction on Circuits and Systems I, Fundamental theory and application, Vol.47, no.3, pp.394-397, Mar. 2000.

[12] Stefano Vitali and Gianluca Setti, "Improving PA Efficiency by Chaos based Spreading in MC/DS-CDMA Systems", Proc. of IEEE ISCAS, pp.1195-1198, Oct. 2006.

[13] A.Abel. and W. Schwarz, "Chaos communication-principles schemes and system analysis", Proc. IEEE Vol.90, no.5, pp.691-710, 2002

[14] M.P.Kennedy, R. Rovatti, and G. Setti, "Chaotic Electronics in Telecommunications. Boca Raton”, CRC, 2000.

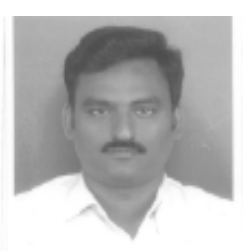

V.Nagarajan received the Bachelors Degree in Electronics and Communication Engineering from Madras University in 1999. He completed his Masters degree in Communication system from Pondicherry University in 2002 . He is pursuing research in the area of wireless communication. $\mathrm{He}$ has published four international journals and presented 10 papers in international and national conferences in the same area. His areas of interest include signal processing and mobile communication. He is a life member of the Institute of Electronics and Telecommunication Engineers (IETE) and Indian Society for Technical Education (ISTE).

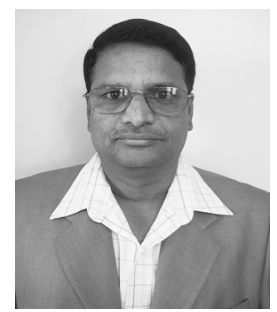

P. Dananjayan received Bachelor of Science from University of Madras in 1979, Bachelor of Technology in 1982 and Master of Engineering in 1984 from the Madras Institute of Technology, Chennai and Ph.D. degree from Anna University, Chennai in 1998. He is working as Professor in the Department of Electronics and Communication Engineering, Pondicherry Engineering College, Pondicherry, India. $\mathrm{He}$

is also a visiting professor to AIT, Bangkok. He has more than 80 publications in National and International Journals. He has also presented more than 150 papers in National and International Conferences. He has guided $7 \mathrm{Ph} . \mathrm{D}$ candidates and is currently guiding $8 \mathrm{Ph} . \mathrm{D}$ students. His research interests include spread spectrum techniques and wireless communication, wireless adhoc and sensor networks. 